\title{
Characterization and Functional Annotation of Uncharacterized ABC Transporter ATP- Binding Protein Rv0986 of Mycobacterium tuberculosis (Strain ATCC 25618 / H37Rv)
}

\author{
Abu Saim Mohammad Saikat ${ }^{1, *}$ \\ ${ }^{1}$ Department of Biochemistry and Molecular Biology, Bangabandhu Sheikh Mujibur Rahman \\ Science and Technology University, Gopalganj, Bangladesh \\ *Correspondence: asmsaikat.bmb@gmail.com
}

\begin{abstract}
The most significant ancient infectious disease tuberculosis is causes by a human pathogen, Mycobacterium tuberculosis (MTB). Amazingly, tuberculosis (TB) has become one of the major causes of human death worldwide. The protein Rv0986 is associated with the ATP-binding cassette domain of the transporters involved in the export of lipoprotein and macrolide, and cell division protein, therefore, related to mycobacterial infection. But the protein Rv0986 is not yet explored. As a result, identification, characterization, and functional annotation of uncharacterized protein Rv0986 were predicted where the structure modeling was generated by using Modeller, Phyre2, and Swiss Model with the structural quality assessment by Ramachandran Plot (PROCHECK), Verify 3d, and Swiss-Model Interactive Workplace as well. Z-scores obtained from Prosa-web were also applied for overall 3D model quality. This in-silico method will uncover the significance of undiscovered uncharacterized protein Rv0986 present in MTB, and indeed it can accelerate the way to enrich our knowledge in the pathogenesis and drug-targeting opportunity against infection by MTB.
\end{abstract}

Keywords: Mycobacterium tuberculosis, Protein Rv0986, Homology Modeling, Ligand binding site, Tuberculosis

\section{Introduction}

Mycobacterium tuberculosis (MTB) is a Gram-positive, acid-fast, and rod-like organism which causes tuberculosis (TB). TB is one of the top lethal communicable diseases (ranking above HIV/AIDS) worldwide. It spreads from infected people with TB banish bacteria into the air; for example, by coughing. Normally, it affects the lungs (pulmonary TB) as well as other sites of the body (extrapulmonary TB). It is estimated that around 10.0 million (range: 9.0-11.1 million) people are in new TB cases and 1.2 million (range, 1.1-1.3 million) TB deaths among HIVnegative people in 2018, resulting in a high risk of TB disease development and spreading globally [1] [2] [3]. The uncharacterized ABC transporter ATP-binding protein Rv0986 of Mycobacterium tuberculosis (strain ATCC 25618 / H37Rv) is the ATPase catalytic subunit of an $\mathrm{ABC}$ transporter complex responsible for coupling the energy of ATP hydrolysis to the import of 
one or more from a variety of substrates including lipoproteins, macrolide, and hemin, such as lipoprotein-releasing system ATP-binding protein LolD thus enhancing mycobacterial infection [4][5][6]. However, the structure of the protein Rv0986is not reported yet. The detailed physicochemical characterization and putative structure with ligand binding active sites are not elucidated, therefore, the In Silico 3D structure prediction of Rv0986 present in MTB with characterization and functional annotation of the protein is proposed by applying in silico structure modeling.

\section{Methodology}

\subsection{Retrieval of Target Amino Acid Sequence}

The amino acid sequence of uncharacterized ABC transporter ATP-binding protein Rv0986 (strain ATCC 25618 / H37Rv) was obtained from UniProtKB [7] with the accession ID P9WQK1. As the 3D structure is unavailable in the Protein Data Bank (PDB), modeling of this unexplored protein was undertaken to utilize a 248 amino acid long sequence of protein Rv0986 present in MTB.

\subsection{Physicochemical Characterization}

The physicochemical properties of the retrieved sequence were determined using two web-based servers. The ProtParam tool of ExPasy [8] employed for the prediction of amino acid composition, instability and aliphatic index, extinction coefficients, and grand average of hydropathicity (GRAVY). Theoretical isoelectric point (pI) was also calculated using the Sequence Manipulation Suite (SMS) version 2 [9]. For the domain prediction of the protein Rv0986, the Conserved Domain (CD) Search Service tool of the National Center for Biotechnology Information (NCBI) [10] was used; and for the determination of motif ScanProsite Tool [11] was applied.

\subsection{Secondary Structure Prediction}

The self-optimized prediction method with alignment (SOPMA) [12] and SPIPRED [13] program was used to predict the secondary structure of Uncharacterized protein Rv0986 Disorder prediction was performed using the DISOPRED tool [14].

\subsection{Structure Modeling and Validation}

As there is no experimentally concluded tertiary structure available for ABC transporter ATPbinding protein Rv0986 (strain ATCC 25618 / H37Rv) of Mycobacterium tuberculosis in the Protein Data Bank (PDB), homology structure modeling was done using three programs including Modeller with HHpred tool, Phyre2, and Swiss-Model server. The designed 3D models 
generated from Modeller, Phyre2, and Swiss-Model were compared and the most suitable 3D model was selected for the final validation. The final modeled structure (3D) was validated using Ramachandran plot analysis (PROCHECK) and the Verify 3D (https://servicesn.mbi.ucla.edu/Verify3D/). The Swiss-Model Interactive Workplace ( https://swissmodel.expasy.org/assess) was applied for the final 3D model quality assessment. Zscores derived from the Prosa-web were applied for the overall 3D model quality validation as well.

\section{Results and Discussion}

\subsection{Physicochemical Characterization}

The amino acid sequence of the protein Rv0986 present in MTB was retrieved in FASTA format and used as a query sequence for the determination of physicochemical parameters. The instability index of the protein Rv0986 of MTB is $32.44(<40)$ indicates the stable nature of the protein [15]. The protein is acidic (pI 5.63, 5.72*) with a molecular weight of 27373.11 Da. Higher aliphatic index values (95.52) of the query protein suggests as a positive factor for increased thermos-stability for a wide temperature range [16]. The hydrophilic nature of the protein and the possibility of better interaction with water [17] were indicated by the lower grand average of hydropathicity (GRAVY) indices value (-0.265) as shown in table 1.

Table 1: Physicochemical Parameters Computed Using ProtParam and SMS Tool

\begin{tabular}{|l|l|}
\hline Physicochemical Parameters & Values \\
\hline Number of amino acids & 248 \\
\hline Molecular weight & 27373.11 \\
\hline Theoretical isoelectric point (pI) & $5.63,5.72 *$ \\
\hline Aliphatic index & 95.52 \\
\hline The instability index (II) & 32.44 \\
\hline Total number of negatively charged residues (Asp + Glu) & 30 \\
\hline Total number of positively charged residues (Arg + Lys) & 26 \\
\hline Grand average of hydropathicity (GRAVY) & -0.265 \\
\hline *pI determined by SMS Version2 & \\
\hline
\end{tabular}

\subsection{Secondary Structure Prediction}

The default parameters (window width: 17; similarity threshold: 8; division factor: 4) were considered by SOPMA for the secondary structure prediction. Utilizing 248 proteins (subdatabase) and 33 aligned proteins, SOPMA predicted 32.66 percent of residues as random coils in comparison to alpha-helix (47.18\%), extended strand (14.92\%) and Beta turn $(5.24 \%)$ as shown in table 2. PSIPRED showing the higher confidence of the prediction of the helix, strand, and coil (Fig.1). 
Table 2: Secondary Structure Elements by SOPMA

\begin{tabular}{|l|l|}
\hline Secondary Structure Elements & Values $\mathbf{( \% )}$ \\
\hline Alpha helix $(\mathrm{Hh})$ & 47.18 \\
\hline $3_{10}$ helix $(\mathrm{Gg})$ & 0.00 \\
\hline Pi helix $(\mathrm{Ii})$ & 0.00 \\
\hline Beta bridge $(\mathrm{Bb})$ & 0.00 \\
\hline Extended strand (Ee) & 14.92 \\
\hline Beta turn (Tt) & 5.24 \\
\hline Bend region $(\mathrm{Ss})$ & 0.00 \\
\hline Random coil $(\mathrm{Cc})$ & 32.66 \\
\hline Ambiguous states & 0.00 \\
\hline Other states & 0.00 \\
\hline
\end{tabular}

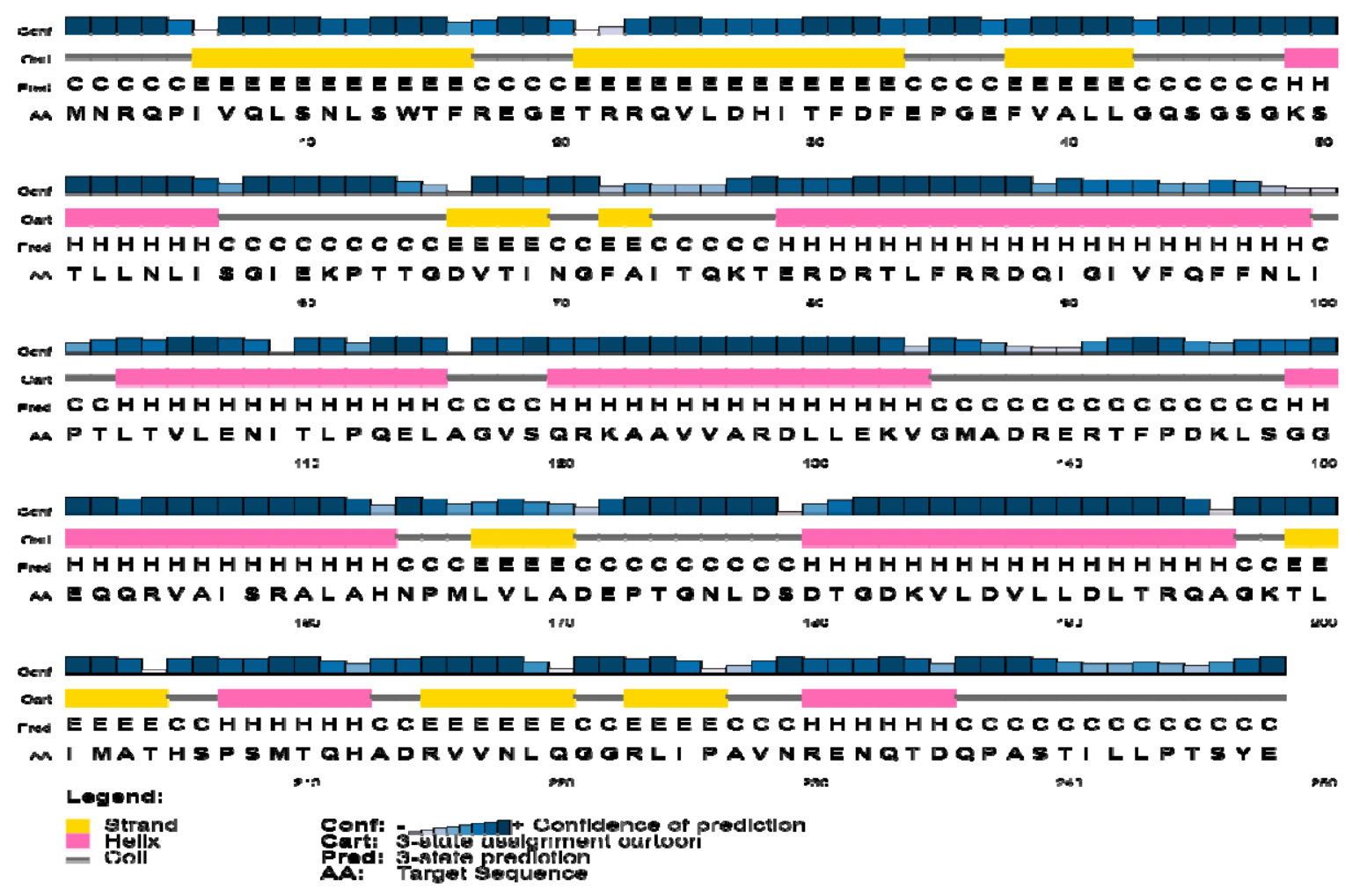

Figure 1 Secondary Structure Prediction by SPIPRED

\subsection{Protein-Protein and Protein-Polynucleotide Binding Sites}


Binding sites were predicted using predict protein server, where 11 different protein binding sites were identified at positions viz.: 1-4, 17-22; 61-62; 84; 87-88; 120-122; 132-133; 139-140; 193; $212 ; 229-234 ; 246$, and 4 different polynucleotide binding sites were identified at positions viz.: 45; 47; 49; and 49-50 (fig. 2).

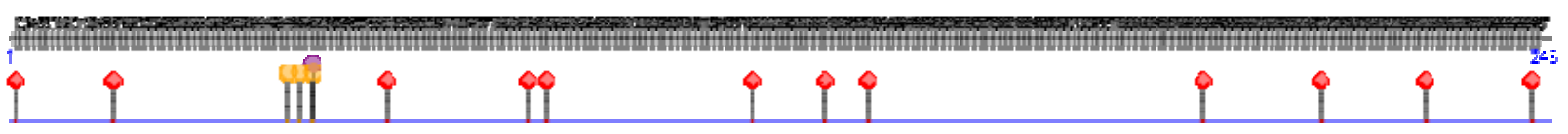

Figure 2 Protein-Protein and Protein-Polynucleotide Binding Sites

\subsection{Structure Modeling and Validation}

The target sequence of the protein Rv0986 present in MTB in FASTA format was inserted to HHpred Template Selection tool [18] as input and the most effective template was selected (3TUI_D) among the number of hits of 250 with the probability rate of 100 percent, E-Value of 6.6e-32, SS of 27.5, Cols of 239 and the target length of 366 (data are not shown) and finally saved the PDB format after forwarding the submitted file to Modeller [19] (Fig. 3). The 3D structure assessment was determined by Ramachandran Map (PROCHECK) showed (Table 3) that $95.2 \%$ of the total residues were found in the core $[\mathrm{A}, \mathrm{B}, \mathrm{L}] ; 4.8 \%$ of residues were in the additional allowed regions [a,b,l,p]; and there was no residue in the both generously allowed regions $[\sim a, \sim b, \sim 1, \sim p]$ and in the disallowed regions (fig. 5). The number of non-glycine and nonproline residues was 209 which was $100 \%$; the end-residues (excl. Gly and Pro) were 2; the glycine residues and proline residues were 18 and 11, respectively, among the total residues(Fig. 3). The 3D model quality assessment was determined by assessment tools to Verify 3D. The 3D model passed this assessment experiment and scored $85.83 \%$ where the minimum score to pass is $80 \%$ (data are not shown). The 3D model assessment was also determined by Swiss-Model Interactive Workplace which validated this model as the MolProbity Score was 3.02 and Ramachandran favored was 97.48\% with the QMEAN (Qualitative Model Energy Analysis), C $\beta$, All Atom, solvation, and torsion values of $-1.15,-2.72,-3.06,0.18$, and -0.61 , respectively (data are not shown). 


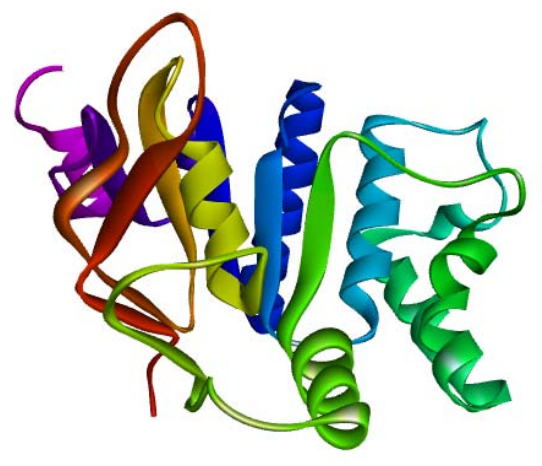

Figure 3 Structure of Rv0986 Predicted by Modeller

Similarly, the 3D model of the protein Rv0986 was performed with Phyre2 based on the most suitable template (c5ws4A_) with the value of confidence of $100.0 \%$ and coverage of $99 \%$ (Fig. 4 -a). 245 residues ( $99 \%$ of the protein sequence) have been modeled with $100.0 \%$ confidence by the single highest scoring template with Phyre2. Secondary structure prediction by Phyre2 was described as the disordered of $16 \%$, alpha-helix of $39 \%$, and beta-strand of $25 \%$ (data are not shown). The 3D structure assessment was performed by Ramachandran Map (PROCHECK) which showed $87.9 \%$ of the residues in most favored regions; $11.2 \%$ were in additional allowed regions; $0.5 \%$ were in disallowed regions, and there was $0.5 \%$ residue in the generously allowed regions (Table 3). The 3D model quality assessment was determined by assessment tools to Verify 3D that showed the 3D model passed this assessment experiment and scored $76.42 \%$ where the minimum score to pass the quality assessment was $80 \%$ (data are not shown).

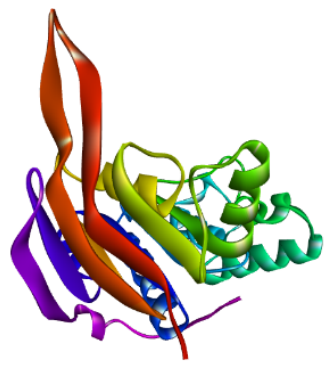

Figure 4 (a): Structure of Rv0986 Predicted by Phyre 2

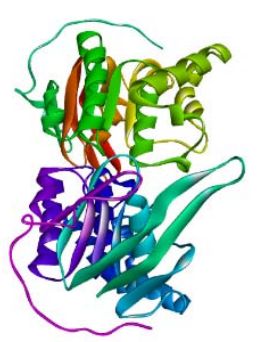

Figure 4 (b): Structure of Rv0986 Predicted by Swiss Model 
The 3D model assessment was also determined by Swiss-Model Interactive Workplace which validated this model as the MolProbity Score was 2.85 and Ramachandran favored of $91.80 \%$ with the QMEAN, C $\beta$, all-atom, solvation, and torsion values of $-1.92,-1.81,-1.88,0.54$, and 1.81 , respectively (data are not shown) that validated the predicted $3 \mathrm{D}$ structure of the protein Rv0986.

Table 3: Ramachandran Plot Analysis

\begin{tabular}{|l|l|l|}
\hline Server & Ramachandran Plot Calculation & Value (\%) \\
\hline \multirow{5}{*}{ Modeller } & Residues in most favored regions [A,B,L] & 95.2 \\
\cline { 2 - 3 } & Residues in additional allowed regions [a,b,l,p] & 4.8 \\
\cline { 2 - 3 } & Residues in generously allowed regions [ a, $\sim \mathrm{b}, \sim 1, \sim \mathrm{p}]$ & 0.0 \\
\cline { 2 - 3 } & Residues in disallowed regions & 0.0 \\
\hline \multirow{5}{*}{ Phyre2 } & Residues in most favored regions [A,B,L] & 87.9 \\
\cline { 2 - 3 } & Residues in additional allowed regions [a,b,l,p] & 11.2 \\
\cline { 2 - 3 } & Residues in generously allowed regions [ a, $\sim \mathrm{b}, \sim 1, \sim \mathrm{p}]$ & 0.5 \\
\cline { 2 - 3 } & Residues in disallowed regions & 0.5 \\
\hline \multirow{5}{*}{ Swiss Model } & Residues in most favored regions [A,B,L] & 92.9 \\
\cline { 2 - 3 } & Residues in additional allowed regions [a,b,l,p] & 6.4 \\
\cline { 2 - 3 } & Residues in generously allowed regions [ a, b, b l, p] & 0.5 \\
\cline { 2 - 3 } & Residues in disallowed regions & 0.2 \\
\hline
\end{tabular}

The 3D model of the protein Rv0986 was also executed with Swiss-Model based on the top five suitable templates (5lj6.1.A, 5lil.1.B, 5li1.1.A, 5lil.1.B, and 2ouk.5.A ) and the target sequence was selected based on the Qualitative Model Energy Analysis (QMEAN) score (-1.03), Global Model Quality Estimate (GMQE) score of 0.73, percentage of sequence identity of 39.50, and the coverage of $96 \%$. The model was saved in PDB format when it was generated. The 3D structure assessment was determined by Ramachandran Map (PROCHECK) showed (Table 3) that $92.9 \%$ of the total residues were found in the core $[\mathrm{A}, \mathrm{B}, \mathrm{L}] ; 92.9 \%$ of residues were in the additional allowed regions $[\mathrm{a}, \mathrm{b}, 1, \mathrm{p}]$; and there was $0.5 \%$ of residue in the generously allowed 
regions $[\sim \mathrm{a}, \sim \mathrm{b}, \sim 1, \sim \mathrm{p}]$ and $0.2 \%$ residue was in the disallowed regions. The number of nonglycine and non-proline residues was 423 which was 100\%; the end-residues (excl. Gly and Pro) were 4; the glycine residues and proline residues were 36 and 22, respectively, among the total residues (Fig. 4-b). The 3D model quality assessment was determined by assessment tools to Verify 3D. The 3D model passed this assessment experiment and scored $78.56 \%$ where the minimum score to pass is $80 \%$ (data are not shown). The 3D model assessment was also determined by Swiss-Model Interactive Workplace which validated this model as the MolProbity Score was 1.53 and Ramachandran favored was 96.88\% with the QMEAN (Qualitative Model Energy Analysis), C $\beta$, All Atom, solvation, and torsion values of -1.03, -2.87, -1.07, 0.10, and 0.53, respectively (data are not shown). The modeled structures of the protein Rv0986 were validated by another structure validation server, Prosa-web [20]. Standard bond angles in the modeled 3D structures were determined by the Prosa-web. Z-score was used to estimate the 'degree of nativeness' of the predicted structures. Z-score for the modeled 3D structure from Modeller, Phyre2, and Swiss-Model were -7.42, -7.31, and -7.37, respectively. In this paper, all three i.e. Modeller, Phyre2, and Swiss-Model servers are presenting similar values that validated the $3 \mathrm{D}$ structures.

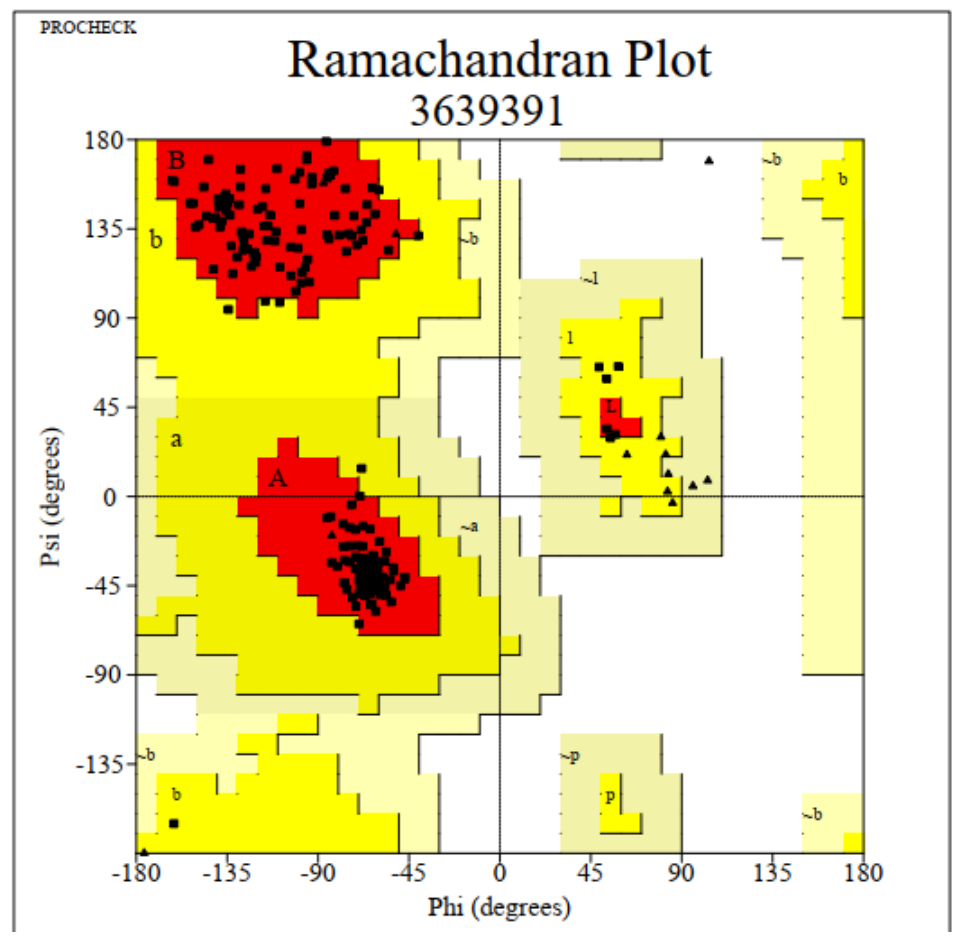

Figure 5 Ramachandran Plot Analysis

\section{Conclusion}

In this study, it is concluded that the structural model of the protein Rv0986 of MTB with predicted active sites for ligand binding through in silico approach where the position of amino acid in the favored region by evaluating the structure. The physicochemical parameters prediction and functional annotation are useful for understanding the action of this protein's 
activity. The homology-modeled protein with its predicted subcellular location in the MTB cell provides insights into the functional role of the protein Rv0986 in pathogenesis which will help to design potential therapeutic drugs against the protein present in MTB.

\section{Acknowledgments}

I would like to thank the Department of Biochemistry and Molecular Biology of Bangabandhu Sheikh Mujibur Rahman Science and Technology University, Gopalganj, Bangladesh for providing a computational platform for completing this project.

\section{Declarations}

Funding: There was no funding received for this manuscript.

Conflict of interest: The author declares no conflict of interest.

\section{References}

[1] WHO | Global tuberculosis report 2019, (n.d.). https://www.who.int/tb/publications/global_report/en/ (accessed March 10, 2020).

[2] A.L. Ellison, EXPLORING THE ROLE OF THE STRINGENT RESPONSE IN by, (2018).

[3] T.A. Duarte, J.S. Nery, N. Boechat, S.M. Pereira, V. Simonsen, M. Oliveira, M.G.M. Gomes, C. Penha-Gonçalves, M.L. Barreto, T. Barbosa, A systematic review of East African-Indian family of Mycobacterium tuberculosis in Brazil, Brazilian J. Infect. Dis. 21 (2017) 317-324. https://doi.org/10.1016/j.bjid.2017.01.005.

[4] C. Wu, S. Chakrabarty, M. Jin, K. Liu, Y. Xiao, Insect ATP-binding cassette (ABC) transporters: Roles in xenobiotic detoxification and Bt insecticidal activity, Int. J. Mol. Sci. 20 (2019). https://doi.org/10.3390/ijms20112829.

[5] K.A. Holland, I.B. Holland, Adventures with ABC-proteins: Highly conserved ATPdependent transporters, Acta Microbiol. Immunol. Hung. 52 (2005) 309-322. https://doi.org/10.1556/AMicr.52.2005.3-4.4.

[6] K. Beis, Structural basis for the mechanism of ABC transporters, Biochem. Soc. Trans. 43 (2015) 889-893. https://doi.org/10.1042/BST20150047.

[7] U.C.-N. acids research, undefined 2019, UniProt: a worldwide hub of protein knowledge, Academic.Oup.Com. (n.d.). https://academic.oup.com/nar/articleabstract/47/D1/D506/5160987 (accessed March 24, 2020).

[8] E. ProtParam, ExPASy-ProtParam tool, (2017).

[9] L. Martin, Genomics and transcriptomics of the molting gland (Y-organ) in the blackback land crab, Gecarcinus lateralis, (2016). https://mountainscholar.org/handle/10217/176669 
(accessed March 24, 2020).

[10] CDD/SPARCLE: the conserved domain database in 2020, Academic.Oup.Com. (n.d.). https://academic.oup.com/nar/article-abstract/48/D1/D265/5645006 (accessed March 24, 2020).

[11] E. De Castro, C. Sigrist, ... A.G.-N. acids, undefined 2006, ScanProsite: detection of PROSITE signature matches and ProRule-associated functional and structural residues in proteins, Academic.Oup.Com. (n.d.). https://academic.oup.com/nar/articleabstract/34/suppl_2/W362/2505523 (accessed March 24, 2020).

[12] NPS@: network protein sequence analysis, Cell.Com. (n.d.). https://www.cell.com/trends/biochemical-sciences/fulltext/S0968-0004(99)01540-6 (accessed March 24, 2020).

[13] D.J.-J. of molecular biology, undefined 1999, Protein secondary structure prediction based on position-specific scoring matrices, Elsevier. (n.d.). https://www.sciencedirect.com/science/article/pii/S0022283699930917 (accessed March 24, 2020).

[14] T. Vikram, P.K.- Bioinformation, undefined 2018, Analysis of Hepatitis E virus (HEV) X-domain structural model, Ncbi.Nlm.Nih.Gov. (n.d.). https://www.ncbi.nlm.nih.gov/pmc/articles/PMC6143357/ (accessed March 24, 2020).

[15] B. Vijay, B. Reddy, K. Guruprasad, B.V.B. Reddy, M.W. Pandit, Correlation between stability of a protein and its dipeptide composition: A novel approach for predicting in vivo stability of a protein from its primary sequence Madhusudan Waman Pandit Centre for Cellular and Molecular Biology Correlation between stability of a protein and its dipeptide composition: a novel approach for predicting in vivo stability of a protein from its primary sequence, Protein Eng. 4 (1990) 155-161. https://doi.org/10.1093/protein/4.2.155.

[16] S. Gill, P.V.H.-A. biochemistry, undefined 1989, Calculation of protein extinction coefficients from amino acid sequence data, Elsevier. (n.d.). https://www.sciencedirect.com/science/article/pii/0003269789906027 (accessed March 24, 2020).

[17] A. Ikai, Thermostability and Aliphatic Index of Globular Proteins, 1980. https://academic.oup.com/jb/article-abstract/88/6/1895/773432 (accessed March 24, 2020).

[18] L. Zimmermann, A. Stephens, S.Z. Nam, D. Rau, J. Kübler, M. Lozajic, F. Gabler, J. Söding, A.N. Lupas, V. Alva, A Completely Reimplemented MPI Bioinformatics Toolkit with a New HHpred Server at its Core, J. Mol. Biol. 430 (2018) 2237-2243. https://doi.org/10.1016/j.jmb.2017.12.007.

[19] B. Webb, A. Sali, Comparative Protein Structure Modeling Using MODELLER, Curr. Protoc. Protein Sci. 86 (2016) 2.9.1-2.9.37. https://doi.org/10.1002/cpps.20.

[20] M. Wiederstein, M.S.-N. acids research, undefined 2007, ProSA-web: interactive web service for the recognition of errors in three-dimensional structures of proteins, 
bioRxiv preprint doi: https://doi.org/10.1101/2020.05.23.112680; this version posted June 17, 2020. The copyright holder for this preprint (which was not certified by peer review) is the author/funder, who has granted bioRxiv a license to display the preprint in perpetuity. It is made available under aCC-BY-ND 4.0 International license.

Academic.Oup.Com. (n.d.). https://academic.oup.com/nar/articleabstract/35/suppl_2/W407/2920938 (accessed March 14, 2020). 